\title{
Anaplastic Oligodendroglioma: A New Treatment Paradigm and Current Controversies
}

\author{
Roth, P ; Wick, W ; Weller, M
}

\begin{abstract}
OPINION STATEMENT: Anaplastic oligodendroglial tumors have gained increasing interest with the emerging role of molecular markers and systemic chemotherapy during the past years. The long-term results of two landmark trials, RTOG 9402 and EORTC 26961, have resulted in a reconsideration of the appropriate therapeutic approaches for patients with these tumors. Both trials indicate that patients whose tumors harbor a 1p/19q co-deletion benefit particularly from the addition of procarbazine/lomustine (CCNU)/vincristine (PCV) chemotherapy to radiation therapy (RT). The median survival of patients with co-deleted tumors treated within the RTOG trial with PCV before irradiation was 14.7 years compared with 7.3 years of patients who received RT alone. Median overall survival has not been reached in the RT plus PCV arm of the EORTC trial, but a similar difference can be anticipated after a follow-up of more than 12 years. In contrast, no such benefit was observed for patients with tumors lacking 1p/19q co-deletion. Outside clinical trials, patients with anaplastic oligodendroglial tumors, and 1p/19q co-deletion therefore should be offered a combined treatment modality regimen, including radioand chemotherapy. PCV, however, is associated with significant hematological toxicity and also nonhematological side effects, which probably translate into reduced quality of life for long-term survivors. Therefore, it might be warranted to replace PCV by temozolomide, which displays a more favorable side effect profile. Data from the NOA-04 study suggest that PCV and temozolomide have similar effects. However, long-term data on the benefit from temozolomide are lacking, making a definite answer on the equivalence of temozolomide and PCV in anaplastic oligodendroglioma (AO) impossible. The current evidence precludes RT alone for AO patients. Neither the RTOG nor the EORTC trial defined the role of chemotherapy alone. A comparison of combined modality treatment with chemotherapy alone followed by RT at progression is pending. Long-term follow-up of NOA-04 patients and results from future trials may help to clarify these questions. With more and more AO patients living 10 years or more, particular attention must be paid to late side effects, such as neurotoxicity, and careful monitoring is required for all treated patients.
\end{abstract}

DOI: https://doi.org/10.1007/s11864-013-0251-7

Posted at the Zurich Open Repository and Archive, University of Zurich

ZORA URL: https://doi.org/10.5167/uzh-79909

Journal Article

Accepted Version

Originally published at:

Roth, P; Wick, W; Weller, M (2013). Anaplastic Oligodendroglioma: A New Treatment Paradigm and Current Controversies. Current Treatment Options in Oncology, 14(4):505-513.

DOI: https://doi.org/10.1007/s11864-013-0251-7 
Patrick Roth ${ }^{1}$, Wolfgang Wick ${ }^{2}$, Michael Weller ${ }^{1}$ *

Affiliations: ${ }^{1}$ Department of Neurology, University Hospital Zurich, Switzerland,

${ }^{2}$ Department of Neurooncology, Neurology Clinic and National Center for Tumor Disease, University of Heidelberg, Germany

*Correspondence: Dr. Michael Weller, Department of Neurology, University Hospital Zurich, Frauenklinikstrasse 26, 8091 Zurich, Switzerland, Tel.: +41 (0)44 255 5500, Fax: +41 (0)44 255 4380, E-mail: michael.weller@usz.ch

Keywords: Anaplastic oligodendroglioma, irradiation, chemotherapy, PCV, temozolomide 


\section{Opinion paragraph}

Anaplastic oligodendroglial tumors have gained increasing interest with the emerging role of molecular markers and systemic chemotherapy during the last years. The long-term results of two landmark trials, RTOG 9402 and EORTC 26961, have resulted in a reconsideration of the appropriate therapeutic approaches for patients with these tumors. Both trials indicate that patients whose tumors harbor a $1 \mathrm{p} / 19 \mathrm{q}$ co-deletion benefit particularly from the addition of procarbazine/lomustine(CCNU)/vincristine (PCV) chemotherapy to radiation therapy (RT). The median survival of patients with co-deleted tumors treated within the RTOG trial with PCV before irradiation was 14.7 years compared to 7.3 years of patients who received RT alone. Median overall survival has not been reached in the RT plus PCV arm of the EORTC trial but a similar difference can be anticipated after a follow-up of more than 12 years. In contrast, no such benefit was observed for patients with tumors lacking 1p/19q co-deletion. Outside clinical trials, patients with anaplastic oligodendroglial tumors and $1 \mathrm{p} / 19 \mathrm{q}$ co-deletion should therefore be offered a combined treatment modality regimen including radio- and chemotherapy. PCV, however, is associated with significant hematological toxicity and also non-hematological side effects which probably translate into reduced quality of life in longterm survivors. Therefore, it might be warranted to replace PCV by temozolomide which displays a more favorable side effect profile. Data from the NOA-04 study suggest that PCV and temozolomide have similar effects. However, long-term data on the benefit from temozolomide are lacking making a definite answer on the equivalence of temozolomide and PCV in anaplastic oligodendroligoma (AO) impossible. The current evidence precludes RT alone for AO patients. Neither the RTOG nor the EORTC trial defined the role of chemotherapy alone. A comparison of combined modality treatment with chemotherapy alone followed by RT at progression is pending. Long-term follow-up of NOA-04 patients and results from future trials may help to clarify these questions. With more and more AO patients living 10 years or longer, particular attention must be paid to late side effects such as neurotoxicity and careful monitoring is required in all treated patients.

\section{Introduction}

The group of anaplastic gliomas, also referred to as WHO (World Health Organization) grade III tumors, has been histologically subdivided into different subtypes: anaplastic astrocytoma 
(AA), anaplastic oligoastrocytoma (AOA), anaplastic oligodendroglioma (AO) and anaplastic ependymoma [1]. Anaplastic oligodendroglial tumors are rare, accounting for less than 5\% of all primary brain tumors (cbtrus.org). In contrast to WHO grade II tumors, these neoplasms display histological features of anaplasia such as nuclear atypia as well as enhanced cellularity and increased number of mitotic figures. The MIB1 index as a marker for proliferation is typically higher than 5\%. The current WHO classification for gliomas lacks the inclusion of molecular markers which have gained increasing interest within the last years and have been proven to be of prognostic significance $[2,3]$. This is of particular interest in anaplastic but also WHO grade II gliomas where the inter-observer variability in the subjective interpretation of the histopathological findings is still significant [4]. Oligodendroglial tumors are characterized by the frequent loss of genetic material from the short arm of chromosome 1 (1p) and the long arm of chromosome 19 (19q) [5]. This situation, also referred to as combined $1 \mathrm{p} / 19 \mathrm{q}$ loss or $1 \mathrm{p} / 19 \mathrm{q}$ co-deletion, is due to an unbalanced translocation resulting in the loss of one hybrid chromosome and loss of heterozygosity (LOH) [6]. How these chromosomal aberrations contribute to the pathogenesis of oligodendroglial tumors has not yet been clarified, but mutations of different genes including homolog of the Drosophila gene capicua (CIC) and far-upstream element (FUSE) binding protein (FUBP1) located on these chromosomal fragments may play a critical role [7]. In the absence of radiation therapy or alkylating chemotherapy, 1p/19q co-deletion may not be associated with prolonged progression-free survival [8] indicating that it is not a prognostic biomarker but rather a predictor for favorable responses to radio- or chemotherapy.

\section{Treatment}

The therapeutic management of AO requires a multidisciplinary approach including surgery, irradiation and chemotherapy. The importance of alkylating chemotherapy has recently been confirmed by the long-term results of 2 randomized phase III trials. However, it remains a matter of debate whether irradiation can be deferred and which alkylating chemotherapy regimen is most suitable in terms of efficacy and toxicity.

Surgery 
- Even with RT and improved chemotherapeutic options (see below), surgery has remained the initial therapeutic procedure. Resection allows for a rapid relief from intracranial pressure and neurological symptoms in tumors which are associated with a significant mass effect.

- There is no clear evidence on the beneficial effects of a gross total resection in anaplastic oligodendroglial tumors. However, based on the results of several studies assessing mainly glioblastoma patients it may be concluded that complete resection is also favorable for the outcome with anaplastic gliomas. 5-aminolevulinic acid (5ALA) is used for the intraoperative identification and resection of gliomas. Compared to conventional microsurgery, 5-ALA-based resection resulted in more complete resections of contrast-enhancing tumor which translated into prolonged progressionfree survival [9]. Similarly to many other trials including patients with WHO grade III and IV gliomas, the majority of patients treated within this study had a histological diagnosis of glioblastoma. Only $3 \%$ of the operated tumors were finally diagnosed as anaplastic oligodendroglial gliomas. Therefore, the role of 5-ALA for the resection of AO remains questionable.

- Most metaanalyses addressing the effect of surgical resection versus biopsy include various histological types of malignant gliomas. Overall, resection may improve the quality of life and also increase overall survival [10]. In parallel, surgery-associated mortality has decreased most likely due to the availability of advanced intraoperative imaging techniques and improved perioperative neuro-anesthesiological care.

- The extent of resection should be confirmed by an appropriate imaging technique, typically MRI, not later than $72 \mathrm{~h}$ after the operation. Extent of resection has been identified as a prognostic factor in several large clinical trials [11-13].

- Because of the increasing importance of molecular markers, the availability of tissue specimens is crucial and represents another important aspect of surgery. Patients whose tumors are not eligible for complete resection due to the localization in the brain or other reasons, need to undergo a biopsy for tissue sampling to allow for subsequent histological and molecular profiling.

\section{Radiation therapy}


- Surgery followed by irradiation was the standard of care of anaplastic oligodendroglioma until the early 1990s. Only trials performed during the 1970s examined the effect of radiotherapy alone in patients with malignant gliomas. Histological glioma specification, however, was mostly lacking at that time resulting in a mixture of grade III and IV tumors [14].

- Randomized trials enrolling specifically patients with AO looking at the net effect of irradiation compared to surgery alone have not been performed and probably will never. Still, there is hardly any doubt that radiotherapy is an effective treatment for patients with AO. This assumption is stressed by retrospective analyses of patient cohorts who had postoperative RT or not reporting that irradiation prolonged survival [15].

- External beam RT is usually administered in standard fractions of 1.8-2 Gy to a total dose in the range of 54 to $60 \mathrm{~Gy}$. There are no data to suggest a beneficial effect of doses higher than 60 Gy [16]. Furthermore, whole brain irradiation (WBRT) is not more effective than partial brain treatment. Clinical trials looking specifically at patients with AO are lacking. In contrast to WBRT, focused radiotherapy may be better tolerated and results much less frequently in reduced quality of life [17].

\section{Chemotherapy}

- For many years there was no consensus on the value of adjuvant chemotherapy in patients with anaplastic gliomas. However, it has been recognized more than 25 years ago that anaplastic oligodendroglial tumors are markedly more sensitive to chemotherapy than most other gliomas [18]. Several smaller, uncontrolled trials within the 1990s reported that AO frequently respond to chemotherapy [19]. The most commonly administered regimen within these studies included procarbazine, lomustine (CCNU) and vincristin (PCV).

- The observation that anaplastic oligodendroglial tumors respond well to PCV chemotherapy resulted in the design of 2 similar trials by the Radiation Therapy Oncology Group (RTOG) and the European Organisation for Research and Treatment of Cancer (EORTC). Both studies aimed at evaluating the role of PCV chemotherapy in combination with radiotherapy compared to radiation therapy alone. The RTOG 9402 trial randomly assigned 291 patients with AO or anaplastic mixed oligo- 
astrocytoma to either RT alone or up to four cycles of PCV chemotherapy with subsequent RT [11]. The EORTC 26951 study enrolled 368 patients with pure or mixed anaplastic oligodendrogliomas and randomized them to receive either RT alone or RT followed by up to six cycles of PCV [12].

- Initial results of both studies were published in 2006 after a median follow-up period of 5 years. Both trials had similar outcomes: (i) patients with 1p/19q co-deletion had a longer median survival than patients with non co-deleted tumors. (ii) The progressionfree survival (PFS) time favored the combination of RT and PCV in both studies. (iii) The median overall survival (OS) was not different between the RT only and the combined modality treatment groups, that is, regardless of whether PCV was given before or after RT. (iv) The addition of PCV to irradiation was associated with significant, mostly hematological toxicity.

- Owing to the lack of benefit on overall survival and the considerable toxicity, the addition of PCV to RT was not established as a new standard of care. Of note, most of the patients who experienced tumor progression after sole irradiation received chemotherapy as salvage treatment in both trials. It was believed that this crossover effect from chemotherapy that was applied at progression precluded the translation of prolonged PFS into a difference in OS.

- In 2013, long-term follow-up data from both trials were reported [20, 21]. The longterm results of these 2 trials have now changed the standards of care for patients with pure and mixed anaplastic oligodendroglial tumors. In the RTOG study, there was no difference in median survival when the entire patient cohort was analyzed. However, within the subgroup of patients with co-deleted tumors, PCV plus RT resulted in a median survival of 14.7 months vs. 7.3 months for those who had been treated with RT alone. No difference in median survival was observed for patients with non codeleted tumors. Long-term results of the EORTC trial were obtained after a median follow-up of 140 months. In contrast to the RTOG trial, there was a prolonged median survival when the whole cohort was assessed, regardless of the $1 \mathrm{p} / 19 \mathrm{q}$ status (OS with RT/PCV 42.3 months vs. 30.6 months, $\mathrm{p}=0.18$ ). 80 patients had a $1 \mathrm{p} / 19 \mathrm{q}$ co-deletion. Here, OS was not yet reached in the RT/PCV group vs. 112 months in the RT group. In the subgroup of patients with non co-deleted tumors, no significant difference between the RT and RT/PCV arm was observed.

- Since both trials used different approaches for the sequencing of radio- and chemotherapy, both options are valid. Consideration may be given to the influence of 
pre-radiotherapeutic chemotherapy in responding patients on the target volume. But, as of now, the target volume will be based on the initial postsurgical MRI.

- PCV administration results in considerable side effects with hematological toxicity as the major limitation. Grade 3 or 4 hematological toxicity was observed in $47 \%$ of the patients treated within the EORTC trial [12] and in $65 \%$ of all patients who were enrolled in RTOG 9402 [11]. Only 42\% of the patients treated within RTOG 9402 tolerated all 4 cycles of PCV. Similarly, in EORTC 26951, a median of 3 out of 6 planned cycles of adjuvant PCV was administered. Only $30 \%$ of the patients could receive all scheduled 6 PCV cycles. Nevertheless, despite many patients not receiving the planned chemotherapeutic regimen, the combined treatment modality arm of the study resulted in superior survival as demonstrated by the long-term follow-up.

- Since $1 \mathrm{p} / 19 \mathrm{q}$ co-deletion is more predictive for the clinical course than histology, 2 large randomized, multicenter trials have been set up for patients with anaplastic glioma using the 1p/19q status as inclusion criterion. The CATNON (NCT00626990) study has been established for patients with tumors lacking a 1p/19q co-deletion. This trial is currently enrolling patients. However, based on the recent findings of RTOG 9402 and EORTC 26951, the second intergroup study, named CODEL (NCT00887146), which had been designed for patients with anaplastic gliomas harboring a 1p/19q co-deletion, was put on hold. The trial had been set up with 3 arms comparing RT plus concomitant and adjuvant TMZ with RT alone or TMZ alone. After publication of the long-term results of the RTOG and EORTC studies, the irradiation only arm was no longer regarded appropriate. Whether this trial will be continued following design modifications is still a matter of debate. The trial may be resumed following a replacement of the RT only arm by a regimen including RT plus PCV.

- A major unresolved question is whether RT can be deferred in favor of chemotherapy alone in patients with co-deleted tumors. Whether PCV or temozolomide should be used is another matter of debate. Currently, no data from large prospective trials are available. Therefore, the results of ongoing and future trials need to be awaited.

- The use of PCV had been abandoned by many neuro-oncologists within the last decade because of its significant, mostly hematological toxicity as outlined above. A survey demonstrated that $42 \%$ of neuro-oncologists use temozolomide alone in patients with AO harboring a co-deletion whereas only $8 \%$ use PCV [22]. A similar analysis revealed that the PCV regimen was virtually completely replaced by 
temozolomide among patients with AO who received chemotherapy alone or together with RT [23].

- There is also a debate on the role of vincristine within the PCV regimen. Most likely, it does not cross the blood-brain barrier and may therefore lack activity against gliomas. In contrast, it may exert severe and non-reversible side effects such as neuropathies hampering the quality of life of many patients [24-26]. On the other hand, we do not know, which drug levels in the contrast-enhancing tumor parts may be of benefit for tumor treatment, and the EORTC and RTOG data have been achived with PCV and not PC. Temozolomide, when administered concomitantly during RT and as adjuvant treatment in patients with newly diagnosed glioblastoma, had only to be stopped in $13 \%$ of the patients indicating that such a regimen is much better tolerable than PCV [27]. Furthermore, in contrast to PCV where vincristine requires intravenous application, temozolomide can be administered orally making it a very convenient therapeutic option. Temozolomide, when used in AO patients at relapse after failure of PCV resulted in considerable response rates and promising survival data $[28,29]$. More recent studies applied temozolomide alone in patients with newly diagnosed AO suggesting similar activity than PCV [30]. In contrast, a retrospective analysis assessing the effects of PCV and temozolomide in patients with AO demonstrated prolonged survival of patients treated with PCV with a median survival of 7.6 years compared to 3.3 years in patients who received temozolomide [31].

However, because of the retrospective design of the study, these results may be biased by several confounding factors. Overall, there is no similar evidence for the activity of temozolomide in AO compared to PCV.

- The long-term results of the NOA-04 study may partially help to clarify the issue of PCV vs. temozolomide chemotherapy as well as the option of deferred RT. The study aimed at assessing efficacy and safety of RT or chemotherapy with either PCV or temozolomide in patients with anaplastic gliomas. At tumor progression, patients within the RT arm were treated with chemotherapy and patients who were initially treated with PCV or temozolomide received RT as salvage treatment. The trial revealed that both, initial radio- or chemotherapy, resulted in similar median PFS and OS [13]. Patients with pure and mixed anaplastic oligodendroglial tumors had virtually the same outcome. Prognostic factors confirmed and identified within this trial included age, extent of resection, histology, MGMT promoter methylation status and IDHI mutation status. The study also confirmed the much better tolerability of 
temozolomide. Whether first-line chemotherapy alone results in promising OS times similar to the combination of RT and PCV in RTOG 9402 and EORTC 26951, will only be clarified by a long-term follow up. Since only a minority of patients treated within the NOA-04 trial had co-deleted tumors, it must be anticipated that further trials are needed for a definitive answer to this question.

\section{Other treatments}

- Except for surgery, irradiation and chemotherapy using PCV or temozolomide, no further treatment modalities have been established in patients with $\mathrm{AO}$. Whenever possible, new pharmaceutical agents as well as novel therapeutic approaches such as immunotherapy or gene therapy should be assessed within clinical trials.

- Anti-angiogenic compounds such as the vascular endothelial growth factor (VEGF) inhibitor bevacizumab have shown some activity in glioblastoma patients [32]. Several smaller studies using bevacizumab alone or in combination with other agents in patients with recurrent oligodendroglial tumors suggest that bevacizumab is also active in these tumors [33-36]. However, the definite role of bevacizumab and similar compounds in the treatment of oligodendroglial tumors - just as for all other grade II and III tumors - needs to be defined within the next years. The TAVAREC trial (NCT01164189) which is currently enrolling patients with recurrent anaplastic gliomas without $1 \mathrm{p} / 19 \mathrm{q}$ co-deletion may shed some light on the efficacy of bevacizumab in these tumors.

\section{Diet and lifestyle}

- Currently, there are no data suggesting that diet and lifestyle changes may have any impact on the course of disease in AO patients. 


\section{References and recommended reading}

1. Louis DN, Ohgaki H, Wiestler OD, et al. The 2007 WHO classification of tumours of the central nervous system. Acta Neuropathol 2007, 114:97-109.

2. - Weller M, Pfister SM, Wick W, et al. Molecular neuro-oncology entering clinical practice: a new horizon. Lancet Oncol 2013, in press.

A comprehensive review on molecular markers in neurooncology

3. Erdem-Eraslan L, Gravendeel LA, de Rooi J, et al. Intrinsic molecular subtypes of glioma are prognostic and predict benefit from adjuvant procarbazine, lomustine, and vincristine chemotherapy in combination with other prognostic factors in anaplastic oligodendroglial brain tumors: a report from EORTC study 26951. J Clin Oncol 2013, 31:328-36.

4. van den Bent MJ. Interobserver variation of the histopathological diagnosis in clinical trials on glioma: a clinician's perspective. Acta Neuropathol 2010, 120:297-304.

5. Reifenberger J, Reifenberger G, Liu L, et al. Molecular genetic analysis of oligodendroglial tumors shows preferential allelic deletions on 19q and 1p. Am J Pathol 1994, 145:1175-90.

6. Jenkins RB, Blair H, Ballman KV, et al. A t $(1 ; 19)(q 10 ; p 10)$ mediates the combined deletions of $1 p$ and $19 q$ and predicts a better prognosis of patients with oligodendroglioma. Cancer Res 2006, 66:9852-61.

7. •• Bettegowda C, Agrawal N, Jiao Y, et al. Mutations in CIC and FUBP1 contribute to human oligodendroglioma. Science 2011, 333:1453-5.

This publication describes 2 genes which may contribute to the pathogenesis of oligodendroglial tumors

8. Weller M, Berger H, Hartmann C, et al. Combined 1p/19q loss in oligodendroglial tumors: predictive or prognostic biomarker? Clin Cancer Res 2007, 13:6933-7.

9. Stummer W, Pichlmeier U, Meinel T, et al. Fluorescence-guided surgery with 5aminolevulinic acid for resection of malignant glioma: a randomised controlled multicentre phase III trial. Lancet Oncol 2006, 7:392-401.

10. Tsitlakidis A, Foroglou N, Venetis CA, et al. Biopsy versus resection in the management of malignant gliomas: a systematic review and meta-analysis. J Neurosurg 2010, 112:1020-32.

11. Cairncross G, Berkey B, Shaw E, et al. Phase III trial of chemotherapy plus radiotherapy compared with radiotherapy alone for pure and mixed anaplastic 
oligodendroglioma: Intergroup Radiation Therapy Oncology Group Trial 9402. J Clin Oncol 2006, 24:2707-14.

12. van den Bent MJ, Carpentier AF, Brandes AA, et al. Adjuvant procarbazine, lomustine, and vincristine improves progression-free survival but not overall survival in newly diagnosed anaplastic oligodendrogliomas and oligoastrocytomas: a randomized European Organisation for Research and Treatment of Cancer phase III trial. J Clin Oncol 2006, 24:2715-22.

13. •• Wick W, Hartmann C, Engel C, et al. NOA-04 randomized phase III trial of sequential radiochemotherapy of anaplastic glioma with procarbazine, lomustine, and vincristine or temozolomide. J Clin Oncol 2009, 27:5874-80.

In this randomized trial radiotherapy or chemotherapy achieved comparable results in patients with anaplastic gliomas

14. Walker MD, Alexander E, Jr., Hunt WE, et al. Evaluation of BCNU and/or radiotherapy in the treatment of anaplastic gliomas. A cooperative clinical trial. J Neurosurg 1978, 49:333-43.

15. Gannett DE, Wisbeck WM, Silbergeld DL, Berger MS. The role of postoperative irradiation in the treatment of oligodendroglioma. Int J Radiat Oncol Biol Phys 1994, 30:56773.

16. Chan JL, Lee SW, Fraass BA, et al. Survival and failure patterns of high-grade gliomas after three-dimensional conformal radiotherapy. J Clin Oncol 2002, 20:1635-42.

17. Phillips C, Guiney M, Smith J, et al. A randomized trial comparing 35Gy in ten fractions with 60Gy in 30 fractions of cerebral irradiation for glioblastoma multiforme and older patients with anaplastic astrocytoma. Radiother Oncol 2003, 68:23-6.

18. Cairncross JG, Macdonald DR. Successful chemotherapy for recurrent malignant oligodendroglioma. Ann Neurol 1988, 23:360-4.

19. Cairncross JG, Macdonald DR, Ramsay DA. Aggressive oligodendroglioma: a chemosensitive tumor. Neurosurgery 1992, 31:78-82.

20. • Cairncross G, Wang M, Shaw E, et al. Phase III trial of chemoradiotherapy for anaplastic oligodendroglioma: long-term results of RTOG 9402. J Clin Oncol 2013, 31:33743.

The long-term follow-up of RTOG 9402 suggests that the combination of radiation therapy with PCV chemotherapy results in prolonged survival in patients with $1 p / 19 q$ co-deleted tumors 
21. • van den Bent MJ, Brandes AA, Taphoorn MJ, et al. Adjuvant procarbazine, lomustine, and vincristine chemotherapy in newly diagnosed anaplastic oligodendroglioma: long-term follow-up of EORTC brain tumor group study 26951. J Clin Oncol 2013, 31:344-50.

The long-term follow-up of the EORTC 26951 trial suggests that the combination of radiation therapy with PCV chemotherapy results in prolonged survival in patients with $1 p / 19 q$ codeleted tumors

22. Abrey LE, Louis DN, Paleologos N, et al. Survey of treatment recommendations for anaplastic oligodendroglioma. Neuro Oncol 2007, 9:314-8.

23. Panageas KS, Iwamoto FM, Cloughesy TF, et al. Initial treatment patterns over time for anaplastic oligodendroglial tumors. Neuro Oncol 2012, 14:761-7.

24. Kellie SJ, Barbaric D, Koopmans P, et al. Cerebrospinal fluid concentrations of vincristine after bolus intravenous dosing: a surrogate marker of brain penetration. Cancer 2002, 94:1815-20.

25. Boyle FM, Eller SL, Grossman SA. Penetration of intra-arterially administered vincristine in experimental brain tumor. Neuro Oncol 2004, 6:300-5.

26. Swain SM, Arezzo JC. Neuropathy associated with microtubule inhibitors: diagnosis, incidence, and management. Clin Adv Hematol Oncol 2008, 6:455-67.

27. Stupp R, Mason WP, van den Bent MJ, et al. Radiotherapy plus concomitant and adjuvant temozolomide for glioblastoma. N Engl J Med 2005, 352:987-96.

28. Yung WK, Prados MD, Yaya-Tur R, et al. Multicenter phase II trial of temozolomide in patients with anaplastic astrocytoma or anaplastic oligoastrocytoma at first relapse.

Temodal Brain Tumor Group. J Clin Oncol 1999, 17:2762-71.

29. Chinot OL, Honore S, Dufour H, et al. Safety and efficacy of temozolomide in patients with recurrent anaplastic oligodendrogliomas after standard radiotherapy and chemotherapy. J Clin Oncol 2001, 19:2449-55.

30. Taliansky-Aronov A, Bokstein F, Lavon I, Siegal T. Temozolomide treatment for newly diagnosed anaplastic oligodendrogliomas: a clinical efficacy trial. J Neurooncol 2006, 79:153-7.

31. - Lassman AB, Iwamoto FM, Cloughesy TF, et al. International retrospective study of over 1000 adults with anaplastic oligodendroglial tumors. Neuro Oncol 2011, 13:649-59. A comprehensive retrospective analysis of patients with anaplastic oligodendroglial tumors 32. Friedman HS, Prados MD, Wen PY, et al. Bevacizumab alone and in combination with irinotecan in recurrent glioblastoma. J Clin Oncol 2009, 27:4733-40. 
33. Taillibert S, Vincent LA, Granger B, et al. Bevacizumab and irinotecan for recurrent oligodendroglial tumors. Neurology 2009, 72:1601-6.

34. - Kreisl TN, Zhang W, Odia Y, et al. A phase II trial of single-agent bevacizumab in patients with recurrent anaplastic glioma. Neuro Oncol 2011, 13:1143-50.

This study suggests activity of the VEGF inhibitor bevacizumab in patients with recurrent anaplastic glioma

35. Seystahl K, Wiestler B, Hundsberger T, et al. Bevacizumab alone or in combination with irinotecan in recurrent WHO grade II and grade III gliomas. Eur Neurol 2013, 69:95101.

36. Chamberlain MC, Johnston S. Bevacizumab for recurrent alkylator-refractory anaplastic oligodendroglioma. Cancer 2009, 115:1734-43. 